\title{
A commonsense assessment of Arrow's theorem
}

\author{
Guido Ortona ${ }^{1}$ \\ DOI 10.1515/jheec-2016-0003
}

\begin{abstract}
The usual, pessimistic interpretation of Arrow's General Possibility Theorem (often "Impossibility" in textbooks) is excessive. The impossibility defined by Arrow occurs only in presence of a tie or of a cycle. These cases are rare or very rare, and their presence may be assessed ex post. If they occur it is necessary to resort to a second-best rule, but this two-stage procedure does not induce strategic behavior, nor impeaches the use of the Condorcet rule (in observance of the axioms) in all the others.

The paper conclusions sustain that implementation of modern management systems to government's public institutions should deal with a different behavior used to know at companies. In this respect, the paper high-lights different aspects between companies and public institutions behavior admitting similarities on organizational structure and internal procedures.
\end{abstract}

Keywords:

JEL Classification:
Arrow's Theorem, Social Choice, Condorcet Rule

$D 71, C 15$

\section{Introduction}

\section{What is Arrow's theorem about?}

An innovative theorem has usually far-reaching implications, whatever the science it pertains to; possibly to the point to make somehow obscure the basic meaning of the theorem itself. It is advisable, consequently, to recall what exactly is the theorem de 
cuius about. Let's start with the textbook presentations. A typical one is the following: "If a social decision mechanism satisfies properties 1,2 and 3 , then it must be a dictatorship"2. Another one is "Can there exist sensible rules which would tell us how to rank different states of the world from an ethical point of view if the only information we have relates to individual preferences?" with "no" as the answer ${ }^{3}$. A third one is sufficient to establish the point we will start from: "Given collective rationality and universal domain, is there a collective choice rule, other than majority decision, that satisfies the conditions of the Pareto principle, non-dictatorship and the independence of irrelevant alternatives? We already know, from its violating the condition of collective rationality, that majority decision does not satisfy all the conditions simultaneously". Again, the answer is "no"4.

Obviously, what above corresponds quite faithfully to the original formulation: "If we exclude the possibility of interpersonal comparisons of utility, then the only methods of passing from individual tastes to social preferences which will be satisfactory [i.e. complete and transitive] and which will be defined for a wide range of sets of individual orderings are either imposed or dictatorial" (Arrow, 1963, p.59).

Quite faithfully, but possibly not fully faithfully. The quotations from textbooks refer to a decision (or choice) rule; while Arrow writes of preferences. Is this difference meaningful 5 ? Prima facie, it is: Actually, Arrow writes (1963, p. 106) that "Little [J. of Pol. Ec., 60 (1952) 422 ff.] has argued cogently that a rule for social decision-making is not the same as a welfare judgment. [...] The distinction is well taken". However, he continues as follows: "I would consider that it is indeed a social decision process with which I am concerned. [...] I regard social values as meaning nothing more than social choices." This sentence is not that clear. What does it mean that social values are social choice? If we consider the first part of the sentence, the only sensible interpretation is that Arrow is interested in finding a social decision rule; and that the discussion of welfare judgments, and hence of a social welfare function based on it, is a step towards the definition of that rule. Textbooks are right. Actually, this interpretation is necessary to give sense to the acceptance of Little's objection, while at the same time not modifying the overall conclusion (i.e. the theorem). Arrow is quite adamant on this point (1963, p.106): "My own viewpoint towards this and other ethical problems coincides with that expressed by Popper: 'Not a few doctrines which are metaphysical, and thus certainly philosophical, can be interpreted as hyposthatizations of methodological rules [...] Where Bergson seeks to locate social values in welfare judgments by individuals, I prefer to locate them in the actions taken by society through its rules for making social decisions" (emphasis added). To provide a conclusive quotation, Arrow agrees with Bergson that "the social welfare [must be interpreted] in terms of social decision processes" (1963, p.106-107).

\footnotetext{
2 Varian, 1987, p.532. Property 1 requires the mechanism to be complete, reflexive and transitive; property 2 refers to Pareto efficiency and property 3 to the independence assumption.

${ }^{3}$ Layard and Walters, 1978, p.43.

${ }^{4}$ Hargreaves Heap et al., 1992, p. 209. The violation of collective rationality is the possibility of a Condorcet cycle.

5 This possibility has been suggested by an anonymous referee of a previous version. Mistakenly, as we will see; but this is why I deemed it necessary to write the second part of this section, that I consider quite obvious.
} 
To sum up to this point, we found that Arrow's impossibility refers to the impossibility of a social decision rule. All decision rules fail, if we want them to respect the required axioms.

\section{A critique of the classical interpretation of Arrow's}

\section{theorem}

What does it mean that a rule fails? The failure of a rule may be defined in several ways. Two are of relevance here. The first is that "the rule provides wrong results so often that it is better not to resort to it". The second is "the rule does not apply to some cases, easily recognizable, but it works without errors in other ones." Consider for instance a clinical test aimed at discovering the presence of a given illness. If it fails according to the first definition, it will produce so many false positive or false negative that it is better not to employ that test. But suppose instead that the test performs perfectly in all cases, provided that the subject is less than 65 y.o. Here we have a failure of the second type, and the test may be employed safely, albeit not universally.

Actually, there is a decision rule that respect all the axioms requested by Arrow and fails according to the second definition: the Condorcet rule. As is well known since May, majority rule respects all the axioms (bar, obviously, transitivity); and the Condorcet rule is made of a series of binary comparisons under majority rule, where all the other alternatives are ruled out by the independence assumption, provided that there is transitivity. Hence, a group that is employing the Condorcet rule to make a collective choice will succeed while fully respecting the axioms of the theorem, provided that a cycle does not occur. But the occurrence of the cycle may well be ascertained ex post. If there is no cycle, the rule works. If there is a cycle, but only in this case, a second-best rule is necessary ${ }^{6}$. This rule must not induce a strategic interest to create ties or cycles in order to resort to it, but to comply with this requirement is not difficult. For instance, this is the case of the rule "to draw lots among the tying or cycling alternatives".

In the framework of the theorem, what above implies that we should not require universality; the meaning of this term, however, is not the usual one. There is plenty of attempts to reduce universality through restrictions imposed on the preference orderings of individuals, like single-peakedness; the literature is both large and well known. Instead, what is argued here is that the restriction must regard the result of the social choice, i.e. the social ordering of preferences. The non-universality refers not to the preferences of the subjects, but to a set of specific cases resulting from them.

Let's go back to the example of the clinical test. Suppose that the test work safely not if a person is less than 65 y.o., but only if s/he is exactly 65. Its utility will be strongly reduced. In our case, the problem is to check whether the Condorcet rule is likely to produce or not. If the absence of a Condorcet cycle is a very rare occurrence, a rule that applies only to this case would not be that useful. On the contrary, if the rare occurrence is the presence of Condorcet cycles, the rule would be precious. Fortunately the real state of the world (better, the state of the real world) is usually the latter. If we rule out the

\footnotetext{
${ }^{6}$ Remember that in absence of a cycle the Condorcet rule is immune from strategic voting.
} 
case of committee decisions with an even number of members, where ties may easily occur, failures, i.e. cycles or ties, are highly unlikely ${ }^{7}$.

\section{The probability of the Condorcet cycle}

We know (see Riker, 1982, p.122) that the probability of a cycle increases with the number of subjects and with that of alternatives, and tends to one. But this is true only under the impartial culture assumption, i.e. if all the orderings of preferences occur with the same frequency or probability (according to the context). With the increase in the number of alternatives and (more important here) of subjects this assumption becomes increasingly unlikely ${ }^{8}$.

Let's start from the typical $3 \times 3$ textbook case. It is easy to compute that the probability of a cycle is only 5.6\%. With larger figures the computing of this probability becomes cumbersome, and inextricably so if we abandon the hypothesis of impartial culture. For the sake of both simplicity and realism the discussion will continue on the basis of simulative experiments, made with the program for the simulation of electoral systems ALEX, developed at the Università del Piemonte Orientale in Italy ${ }^{9}$. The program allows to avoid the equiprobability of the orderings through a quite realistic device. The alternatives (parties, in the frame of the program) are ordered (typically, left to right). The user inputs the first preferences, according to assumed shares, and two parameters: the first defines the probability that the second preference is an adjacent party/alternative; the second that the second preference is a second-to-adjacent one. If the sum of the two is lower than 1, the second preference may be another party/preference, at random ${ }^{10}$. The procedure is iterated to produce the third preference, and so on ${ }^{11}$. Specific provisions, not worth discussing here, deal with alternatives at the extremes of the ordering. In the context of this paper a high value of the parameters corresponds to more clustered preferences. Table 1 presents a set of simulations for committee decisions.

\footnotetext{
7 Even the most classical case of cyclicity, that of the divide-the-dollar-like decisions, requires quite peculiar conditions to occur in the real world - not to mention that its exposure to cycling has been plausibly challenged on theoretical grounds (see Rae and Schickler (1997, p.175), and Baron and Ferejohn, 1989). Tullock, 1967, stops here in his critique to the theorem, confirming it but claiming that in the real world cycles are rare.

8 By the way, impartial culture has been proved to be the scenario that maximizes the probability of a cycle. See Tsetsin et al., 2003.

${ }^{9}$ See Bissey et al., 2004 and 2007.

10 If the spatial distance is not defined, the program chooses at random between the alternative on the left and that on the right. If it is defined, it chooses the closest one.

11 The default values (that will be adopted here) are 0.8 and 0.1 (or 0.2 if there are three alternatives).
} 
Table 1 - Simulations for committee decisions

\begin{tabular}{|c|l|l|l|l|l|l|}
\hline $\mathrm{N}$ & $\begin{array}{l}\text { Number of } \\
\text { Subjects }\end{array}$ & $\begin{array}{l}\text { Number of } \\
\text { Alternatives }\end{array}$ & $\begin{array}{l}\text { Share of first } \\
\text { preferences } \\
\text { for ordered } \\
\text { alternatives }\end{array}$ & $\begin{array}{l}\text { Probability that } \\
\text { the second } \\
\text { preference is } \\
\text { adjacent/ } \\
\text { second to } \\
\text { adjacent to the } \\
\text { first }\end{array}$ & $\begin{array}{l}\text { Number of } \\
\text { failures to } \\
\text { provide a } \\
\text { Condorcet } \\
\text { winner }\end{array}$ & $\begin{array}{l}\text { Number of } \\
\text { simulations }\end{array}$ \\
\hline 1 & 3 & 3 & $33.33,33.34,33.33$ & $0.501 / 0.49912$ & 56 & 1000 \\
\hline 2 & 3 & 3 & $33,34,33$ & $0.8 / 0.2$ & 35 & 1000 \\
\hline 3 & 3 & 3 & $43,20,37$ & $0.8 / 0.2$ & 19 & 1000 \\
\hline 4 & 3 & 4 & 25 each & $0.334 / 0.333$ & 109 & 1000 \\
\hline 5 & 3 & 4 & 25 each & $0.8 / 0.1$ & 57 & 1000 \\
\hline 6 & 3 & 4 & $20,30,40,10$ & $0.8 / 0.1$ & 30 & 1000 \\
\hline 7 & 4 & 4 & $20,30,40,10$ & $0.8 / 0.1$ & 400 & 1000 \\
\hline 8 & 5 & 5 & 20 each & $0.251 / 0.249$ & 205 & 1000 \\
\hline 9 & 5 & 5 & $15,35,10,30,10$ & $0.8 / 0.1$ & 97 & 1000 \\
\hline 10 & 5 & 5 & $15,35,10,30,10$ & $0.8 / 0.1$ & 2 & 1000 \\
\hline
\end{tabular}

The first row is the classical textbook case, and provides the theoretical prediction mentioned above. If we move to more realistic clusters of preferences (row 2) the number of cycles decreases; and if we add, as a further element of realism, different shares for the first preferences (row 3 ) their number falls below $2 \%$. As expected, if the number of alternatives is even the probability of a Condorcet winner increases (row 4), but it decreases again if we add clustering (row 5) and different shares of first preferences (row 6). The Condorcet rule fails significantly only if the number of alternatives is small and that of subjects is even (row 7). In row 8, 9 and 10 we may observe that if there is equiprobability the frequency of cycles is close to the expected one, $200^{13}$, but it decreases rapidly if we add clustering of preferences (row 9) and differentiate the location of the alternatives on the left-right axis (row 10) ${ }^{14}$.

When stating his theorem, Arrow was concerned not only with committee decisions, but also with electoral matters (see e.g. Arrow, 1963, p.6), i.e. with a setting with many subjects. Table 2 provides the corresponding simulations ${ }^{15}$.

Table 2 - Simulations for electoral decisions

\begin{tabular}{|l|l|l|l|l|l|l|}
\hline $\mathrm{N}$ & $\begin{array}{l}\text { Number of } \\
\text { Subjects }\end{array}$ & $\begin{array}{l}\text { Number of } \\
\text { Alternatives }\end{array}$ & $\begin{array}{l}\text { Share of first } \\
\text { preferences } \\
\text { for ordered } \\
\text { alternatives }\end{array}$ & $\begin{array}{l}\text { Probability that } \\
\text { the second } \\
\text { preference is } \\
\text { adjacent/ }\end{array}$ & $\begin{array}{l}\text { Number of } \\
\text { failures to } \\
\text { provide a } \\
\text { Condorcet }\end{array}$ & $\begin{array}{l}\text { Number of } \\
\text { simulations }\end{array}$ \\
\hline
\end{tabular}

12 The program requires the first probability to be greater than the second.

13 The (small) difference is arguably due to the rounding of probabilities in column 5 .

${ }^{14}$ In row 10 there is a large distance between parties three and four.

15 To my knowledge what follows is the largest simulative assessment of the probability of failure of the Condorcet procedure so far available. However, failure include also ties, hence its results are not strictly comparable with those of previous simulations on the occurrence of a cycle. The results of most simulations, however, are highly coherent with the ones presented here. See Van Deemen (2014) for a survey. 


\begin{tabular}{|l|l|l|l|l|l|l|}
\hline & & & & & $\begin{array}{l}\text { second to } \\
\text { adjacent to the } \\
\text { first }\end{array}$ & winner \\
& & & & \\
\hline 1 & 100 & 3 & $33,34,33$ & $0.51 / 0.49$ & 216 & 1000 \\
\hline 2 & 100 & 3 & $33,34,33$ & $0.8 / 0.2$ & 4 & 1000 \\
\hline 3 & 200 & 3 & $33,34,33$ & $0.8 / 0.2$ & 0 & 1000 \\
\hline 4 & 100 & 4 & $15,30,35,20$ & $0.8 / 0.1$ & 64 & 1000 \\
\hline 5 & 200 & 4 & $15,30,35,20$ & $0.8 / 0.1$ & 30 & 1000 \\
\hline 6 & 500 & 4 & $15,30,35,20$ & $0.8 / 0.1$ & 1 & 300 \\
\hline 7 & 100 & 5 & $15,35,10,30,10$ & $0.8 / 0.1$ & 77 & 1000 \\
\hline 8 & 300 & 5 & $15,35,10,30,10$ & $0.8 / 0.1$ & 13 & 1000 \\
\hline
\end{tabular}

If we accept impartial culture we observe, as expected, quite a number of failures (row 1 ). But if we introduce the clustering of preferences they almost disappear (row 2); and even more so, interestingly enough, if the number of voters increases (row 3). An even number of parties increases the probability of a failure (row 4), but again the increase of the number of voters reduces it (rows 5 and 6), as happens also with an odd number of voters (rows 7 and 8 ). In actual elections voters are usually much more than 500 , hence we may argue quite safely that in a real-world election the Condorcet cycle should occur only exceptionally.

This is confirmed by tables 3 and 4, which present the simulations for two (stylized) cases taken from the real world: the Lower Chamber of the UK, as representative of majoritarian Parliaments, and that of the Netherlands, as representative of proportional ones.

Table 3 - Simulation with data similar to the real ones of UK polls of $2015^{16}$

\begin{tabular}{|l|l|l|l|l|l|l|}
\hline $\mathrm{N}$ & $\begin{array}{l}\text { Voters } \\
\text { per } \\
\text { district }\end{array}$ & $\begin{array}{l}\text { Number } \\
\text { of } \\
\text { parties }\end{array}$ & $\begin{array}{l}\text { Share of first } \\
\text { preferences } \\
\text { for ordered parties }\end{array}$ & $\begin{array}{l}\text { Probability that } \\
\text { the second } \\
\text { preference is } \\
\text { adjacent/ second } \\
\text { to adjacent to the } \\
\text { first }\end{array}$ & $\begin{array}{l}\text { Number of } \\
\text { failures to } \\
\text { provide a } \\
\text { Condorcet } \\
\text { winner }\end{array}$ & $\begin{array}{l}\text { Number of } \\
\text { simulations }\end{array}$ \\
\hline 1 & 100 & 5 & $\begin{array}{l}4.2,33.2,8.7, \\
40.1,13.8\end{array}$ & $0.251 / 0.24917$ & 11 & 100 \\
\hline 2 & 100 & 5 & $\begin{array}{l}4.2,33.2,8.7,40.1, \\
13.8\end{array}$ & $0.8 / 0.1$ & 4 & 100 \\
\hline 3 & 200 & 5 & $\begin{array}{l}4.2,33.2,8.7,40.1, \\
13.8\end{array}$ & $0.8 / 0.1$ & 1 & 100 \\
\hline 4 & 1000 & 5 & $4.2,33.2,8.7,40.1$, & $0.8 / 0.1$ & 0 & 100 \\
\hline
\end{tabular}

\footnotetext{
16 Regional parties and parties that did not manage to get at least one MP are excluded. Real shares of votes have been corrected accordingly (the total share accounted for here is $91.6 \%$ of the real one). The order assumed, left to right, is Green Party, Labour, Lib-lab, Conservative and UKIP.

17 These parameters imply that the order of preferences is random, bar a small bias due to a constraint of the program (the first parameter must be greater than the second). This holds also for the values of the first row of the following table.
} 
Ties or cycles are rare; more important for real life, their number decreases with the clustering of preferences and the increase of voters.

Table 4 - Simulation with data similar to the real ones of Dutch polls of $2012^{18}$

\begin{tabular}{|l|l|l|l|l|l|l|}
\hline $\mathrm{N}$ & $\begin{array}{l}\text { Voters } \\
\text { per } \\
\text { districts }\end{array}$ & $\begin{array}{l}\text { Number of } \\
\text { parties }\end{array}$ & $\begin{array}{l}\text { Share of first } \\
\text { preferences } \\
\text { for ordered parties }\end{array}$ & $\begin{array}{l}\text { Probability that } \\
\text { the second } \\
\text { preference is } \\
\text { adjacent/ } \\
\text { second to } \\
\text { adjacent to the } \\
\text { first }\end{array}$ & $\begin{array}{l}\text { Number of } \\
\text { failures to } \\
\text { provide a } \\
\text { Condorcet } \\
\text { winner }\end{array}$ & $\begin{array}{l}\text { Number of } \\
\text { simulations }\end{array}$ \\
\hline 1 & 100 & 8 & $\begin{array}{l}10.4,2.5,26.6,3,3 \\
9.1,8.6,28.6,10.9\end{array}$ & $0.143 / 0.142$ & 22 & 100 \\
\hline 2 & 100 & 8 & $\begin{array}{l}10.4,2.5,26.6,3,3 \\
9.1,8.6,28.6,10.9\end{array}$ & $0.8 / 0.1$ & 23 & 100 \\
\hline 3 & 500 & 8 & $\begin{array}{l}10.4,2.5,26.6,3,3 \\
9.1,8.6,28.6,10.9\end{array}$ & $0.8 / 0.1$ & 14 & 100 \\
\hline 4 & 1000 & 8 & $\begin{array}{l}10.4,2.5,26.6,3,3 \\
9.1,8.6,28.6,10.9\end{array}$ & $0.8 / 0.1$ & 4 & 100 \\
\hline 5 & 2000 & 8 & $\begin{array}{l}10.4,2.5,26.6,3,3 \\
9.1,8.6,28.6,10.9\end{array}$ & $0.8 / 0.1$ & 2 & 100 \\
\hline
\end{tabular}

Here the number of parties and the presence of small centrist parties make the absence of a choice more likely for a small number of voters; but again its probability decreases steadily with more realistic figures.

In any case, it must be stressed that the aim of the simulations above was not to show that ties or cycles are so rare that it is not necessary to look for a universal rule; instead, that they are sufficiently rare for a rule that can be implemented if they do not occur to make sense.

\section{A more optimistic interpretation of the theorem}

To sum up. The Condorcet rule is affected by the impossibility proved by Arrow only due to the possibility of a cycle. But the presence of a cycle may be ascertained $a$ posteriori with certainty, and the eventuality of its occurrence does not induce strategic behaviors. Hence the Condorcet rule may be used obeying to the axioms of Arrow whenever there is no cycle. And because the cycles are rare, the rule may be applied commonly. In other words, there is no social choice rule that is always transitive; but

18 Testimonial parties, 50-plus (pensioners) party and parties that did not manage to get at least one MP are excluded. The total share accounted for here is $93.1 \%$ of the real one. The order assumed, left to right, is SP, GL, Pvda, CU, CDA, D66, VVD and PPV. 
there is one, and only one (as proved by May) that very often is; and it may safely used when it is. That rule is the Condorcet rule.

It follows that the typical textbook interpretations of the theorem are unduly pessimistic. It is not true that "three very plausible and desirable features of a social decision mechanism are inconsistent with democracy" (Varian, 1987, p.532), i.e. that "there is, in social life, a tradeoff between social rationality and the concentration of power" (Shepsle and Bonchek, 1997, p.67); nor that the "only possibility" for a social welfare function to satisfy those three conditions "is a dictatorial welfare function" (Jehle and Reny, 1998, p.341). Those features are inconsistent with democracy only in rather peculiar cases, while in most cases a non-dictatorial welfare function allows a social choice that respects the axioms of Arrow. The theorem must be read not as a limitation, but as a vindication of democracy.

\section{References}

Arrow, KJ 1950, 'A Difficulty in the Concept of Social Welfare', Journal of Political Economy, 58, pp. 328-346.

Arrow, KJ 1963, Social Choice and Individual Values, 2nd ed., Yale Un. Press, New Haven.

Baron, DP and Ferejohn, J 1987, 'Bargaining and Agenda Formation in Legislatures', American Economic Review Paper and Proceedings 77, pp. 303-309.

Bissey, ME, Carini, M and Ortona, G 2004, 'ALEX3: a Simulation Program to Compare Electoral Systems', Journal of Artificial Societies and Social Simulation, 7, n.3, pages not numbered.

Bissey, ME and Ortona, G 2007, 'The Program for the Simulation of Electoral Systems ALEX4.1: What it does and how to use it', Polis Working Papers n. 91, Università del Piemonte Orientale, Alessandria, Italy.

Hargreaves Heap, S., Hollis, M., Lyons, B., Sugden, R. and Weale, A. 1992, The Theory of Choice, a Critical Guide, Blackwell, Oxford.

Jehle, GA. and Reny, PJ 1998, Advanced Microeconomic theory, Addison-Wesley, Boston.

Layard, PRG and Walters, AA 1978, Microeconomic theory, McGraw-Hill, London.

May, KO 1952, 'A Set of Independent, Necessary and Sufficient Conditions for Simple Majority Decision', Econometrica, 20, pp. 680-684.

Mueller, DC 2003, Public Choice III, Cambridge Un. Press, Cambridge. 
Rae, DW and Schickler, E 1997, 'Majority Rule', In Mueller, DC, Perspectives on Public Choice, Cambridge Un. Press, Cambridge, pp.163-180.

Riker, WH 1982, Liberalism against Populism, Freeman, London.

Shepsle, KA. and Bonchek MS 1997, Analyzing Politics, Norton, New York and London.

Tsetsin, I, Regenwetter, M and Grofman, B 2003, 'The impartial culture maximizes the probability of majority cycles', Social Choice and Welfare, 21, pp. 387-398.

Tullock, G 1967, 'The General Irrelevance of the General Impossibility Theorem', The Quarterly Journal of Economics, 81, pp. 256-270.

Van Deemen, A 2014, 'On the empirical relevance of Condorcet's paradox', Public Choice, 158, pp. 311-330.

Varian, HR 1987, Intermediate Microeconomics, Norton, New York and London. 